\title{
OUTFLOWS FROM YOUNG STARS: THE ROSETTA STONE OF ASTROPHYSICAL JETS?
}

\author{
T. Ray $^{1}$
}

\begin{abstract}
It can be argued that all astrophysical jets, from lowly substellar objects such as young brown dwarfs to massive black holes at the centre of AGN, are generated by the same basic physical mechanism. While the nature of that mechanism is still debated, jets from young stars may represent our best chance of deciphering it. There are several reasons for this statement. First of all they are nearby, thus affording us not only high spatial resolution studies of the "central engine" but also time-resolved analysis of their kinematics. Moreover as they radiate emission lines, spectroscopy can reveal radial velocities, temperature, density, ion fraction, etc., along their flow. This wealth of data is a challenge to the theorist/computational simulator but also a highly effective means of discriminating between models. In addition, the observations tightly constrain laboratory experiments. Here, I briefly review what is known about conditions in jets from young stars as a guide to experiments, their generation including their link with accretion disks, and their evolution from the earliest proto-stellar to pre-main sequence phase.
\end{abstract}

\section{Introduction}

Jets are seen from a whole host of astrophysical objects ranging from massive black holes at the centre of active galaxies (Marshall et al. 2002), GRBs (Piran 2005), proto-planetary nebulae (Sahai 2002), young stars (Bally et al. 2007) and even brown dwarfs (Whelan et al. 2005). There are good physical reasons for suspecting that the same basic mechanism generates the jet in every case despite the enormous differences in mass (Livio 1999, 2011). Certainly all of these objects have a few basic ingredients in common: a central gravitating source, an accretion disk and magnetic fields that thread through the disk. It is the interplay of these ingredients that is thought to give rise to the jet through magneto-hydrodynamic

${ }^{1}$ School of Cosmic Physics, Dublin Institute for Advanced Studies, 31 Fitzwilliam Place, Dublin 2, Ireland 
(MHD) processes and, in particular, centrifugal ejection (Pudritz et al. 2007; Shu et al. 2000). While such MHD models are generally accepted, it is very difficult to find hard observational support. This is primarily because the physical data on individual jets is usually incomplete. Take for example jets from active galactic nuclei (AGN). These have been known for over a century and have been mapped in exquisite detail (e.g. Sparks et al. 1996). While we can use the intensity and polarisation of this emission to estimate the strength and direction of the magnetic field (Dulwich et al. 2007), we know relatively little about other basic jet parameters such as velocity, mass loss rate or even composition. In comparison, we can deduce an enormous amount of detail about jets from young stars. This is not only because they are relatively nearby and thus we can explore them with high spatial resolution (Ray et al. 2007) but also because they primarily produce line emission unlike jets from active galaxies. Using the ratio of various lines, we can determine such fundamental parameters as the density, temperature and ionisation fraction in a jet (Ray et al. 2007). Moreover, through measuring the shift in the lines, we can determine a jet's radial velocity and by combining this with images taken a few years apart, its full 3-D velocity. Thus jets from young stars could be the ideal touchstone to understand how astrophysical jets in general are generated as they put severe constraints on numerical and laboratory simulations.

\section{Jets from young stars: The basics}

Jets from young stars are one of the most striking phenomenon in astrophysics. Comprehensive reviews are giving by Bally et al. (2007) and Ray et al. (2007) including a number of dramatic images. Here, only some basic properties are listed.

The first point to note is that they can be observed in several forms (ionised, neutral atomic and molecular) and over a wide range of wavelengths from X-rays to radio waves (see below). In the optical regime their emission derives from a number of lines both permitted (e.g. H $\alpha$ ) and forbidden (e.g. [OI] $\lambda 6300)$. Analysis has shown that these are produced in the cooling zone of a radiative shock with typical shock velocities of tens of $\mathrm{km} / \mathrm{s}$. In contrast radial and tangential velocity measurements, from spectroscopy and multi-epoch imaging respectively, suggest jet velocities of at least several hundred $\mathrm{km} / \mathrm{s}$, i.e. close to the expected escape velocity from a young star (Hartigan et al. 2011). Since typical temperatures are around $10^{4} \mathrm{~K}$, i.e. the sound speed in the jet is approximately $10 \mathrm{~km} / \mathrm{s}$, the observed jet velocities are not only larger than the average shock velocity but also highly supersonic. The simplest explanation of this conundrum is that the shocks are internal "working surfaces" caused by relatively small velocity variations in the outflow itself (Raga et al. 2007).

A cursory examination of an optically visible young stellar object (YSO) jet shows that it is highly collimated (with opening angles, $\theta$, of at most a few degrees). Since the Mach number $\mathrm{M}_{\text {jet }} \approx 20-30$, this suggests free expansion, i.e. $\theta$ (radians $) \approx 1 / \mathrm{M}_{\text {jet }}$. In addition to being highly collimated, outflows from young stars stretch for surprisingly large distances of up to several parsecs, i.e. distances 
comparable to the size of the parent molecular cloud. Moreover they may play an important role in star formation by:

Removing angular momentum from the circumstellar disk, thereby allowing accretion to occur (e.g. Pudritz et al. 2007).

Promoting dispersion of the infalling envelope, thus limiting the final star/protoplanetary disk mass (Seale \& Looney 2008).

Injecting turbulence into the surrounding cloud, thereby helping to prevent it from gravitational collapse (Arce et al. 2010).

Of crucial importance is the fact that we can measure a whole host of parameters for YSO jets which in turn provides strong constraints for numerical and laboratory simulations. Typical jet velocities and temperatures have already been mentioned but various line ratios also provide electron density, and, under the assumption of charge exchange, ionisation fraction and total density (Podio et al. 2009). The ionisation fraction, $\chi_{e}$, is typically low, i.e. most of an optically visible jet consists of neutral atomic species, although $\chi_{e}$ varies widely from outflow to outflow and even within an individual outflow. Combining the total density with the jet velocity, $V_{\text {jet}}$, and the jet radius, $r_{\text {jet }}$, one can derive an estimate of the mass loss rate, $\dot{M}_{\text {jet }}$. Typical jet radii are of order 100 AU at a few thousand AU from the source, i.e. they are just about spatially resolved for the nearest star forming regions. Derived mass loss rates depend not only on the mass of the young star, as one might imagine, but also on evolutionary status. Representative values for evolved optically visible YSOs, with masses similar to the Sun, i.e. classical $\mathrm{T}$ Tauri stars, are around $10^{-7} M_{\odot} \mathrm{yr}^{-1}$ (Dougados et al. 2010). Less evolved stars, e.g. so-called Class 0 and Class I sources, tend to have higher mass loss rates (Curtis et al. 2010, and see below Sect. 4) and are optically obscure. This may apply not only to the source but also to the outflow in which case one has to resort to infrared/millimeter emission lines to observe the jet. As these lines are frequently produced from molecular transitions, e.g. the $2.12 \mu \mathrm{m}$ line of $\mathrm{H}_{2}$, such transitions constitute the best tracers of early stage outflows. For example jets from Class 0 sources can be observed in $\mathrm{SiO}$ (Codella et al. 2007) and such observations not only show that these outflows are powerful but also that they are highly collimated even when very young $\left(\sim 10^{4} \mathrm{yrs}\right)$.

Measuring the ratios of various species in YSO jets can also give us clues to their origin. For example if they are launched close to the star $(\sim 0.1 \mathrm{AU})$, i.e. within the so-called dust sublimation zone, they should not contain any signature of dust. This is because all of the refractory species, normally locked up in grains, should have been returned to the gas phase. Detailed spectroscopic analysis of jets show that they have typical abundances of refractory species that are intermediate between the ISM and solar (Podio et al. 2009). Such a result can be understood if, as expected, the weak shocks within jets do not fully destroy the dust grains but instead result in partial sublimation. Moreover when jet material passes through higher velocity shocks, increased sublimation is observed (Podio et al. 2009). 
As already mentioned it is possible to measure jet mass loss rates. This can be done either at extended distances from the YSO or alternatively using outflow tracers at the source itself. The derived values can be compared with the measured accretion rates and although there are numerous uncertainties (Antoniucci et al. 2008 ) it seems mass loss rates are typically a few per cent of accretion rates.

The wealth of knowledge about YSO jets in comparison to AGN jets is striking. In the case of the latter not only is the jet velocity less certain but mass loss rates and accretion rates are poorly known. Moreover the relative importance of the different components in AGN jets (leptons and hadrons) as sources of radiation is even disputed (Sikora 2011)! One saving grace in the case of AGN jets however is that we have some idea of their magnetic field strengths (O'Sullivan \& Gabuzda 2009) assuming energy equipartition between radiating particles and magnetic fields. In contrast (but see below Sect. 6) magnetic fields in YSO jets remain highly uncertain.

\section{The search for jet rotation}

In recent years a number of investigations have been carried out to search for rotation in YSO jets (e.g. Bacciotti et al. 2002; Coffey et al. 2007; Woitas et al. 2005). Why are such studies important? There are two main reasons:

To check whether jets are a means of transporting angular momentum from accreted matter. In order for matter to be accreted onto a YSO, the surplus angular momentum of the material must be removed. It is assumed that this loss occurs in the accretion disk and while instabilities (e.g. the magnetorotational instability or MRI) within the disk may play a role (Salmeron et al. 2007), jet production may also be a way of removing angular momentum.

Assuming jets are found to rotate, then the amount of rotation is a clue from where the jet material was launched (Ferreira et al. 2006). This is of major importance to competing jet-launching models. For example if the jet is launched close to the star/disk co-rotation radius, as assumed by $\mathrm{X}$-wind models (Shu et al. 2000), then the angular momentum transport in the jet is expected to be low. On the other hand if the material comes from say 0.1-1.0 AU as predicted by disk (D)-wind models (Pudritz et al. 2007), the expected rotation velocities should typically be a few percent of the poloidal (on-axis) jet velocity and observable.

In any event, assuming the jet is launched via MHD centrifugal ejection, the magnetic field configuration is related to the accretion rate in the disk and the mass loss rate in the jet. More precisely:

$$
\begin{gathered}
\dot{J}_{W}=\dot{M}_{W} \Omega r_{A}^{2} \\
\dot{J}_{A c c}=\frac{1}{2} \Omega r^{2} \dot{M}_{A c c}
\end{gathered}
$$


where $\dot{J}_{W}, \dot{J}_{A c c}$, and $\dot{M}_{W}, \dot{M}_{A c c}$, are the angular momentum fluxes in the wind/jet and accreted material, and the mass fluxes in the wind/jet and accreted material respectively. $\Omega$ and $r_{A}$ are the angular velocity of the disk and Alfvén radius at the launch radius $r$. Since the jet removes the angular momentum of the accreted material, it follows that:

$$
\frac{\dot{M}_{W}}{\dot{M}_{A c c}}=\frac{1}{2}\left(\frac{r}{r_{A}}\right)^{2} .
$$

In other words, measuring the ratio of the mass loss rate through the jet to the accretion rate gives us a handle on the so-called lever arm $\lambda=r_{A} / r$, i.e. the ratio of the Alfvén to launch radii (Ferreira et al. 2006). As typical mass loss rates through a jet are $1-3 \%$ of the independently measured accretion rate, this suggests $\lambda \approx 4-9$ irrespective of the MHD model.

As mentioned above, a number of studies have now been completed to search for rotation in YSO jets. These observations are very challenging and require not only high spatial resolution (to resolve the jet in the direction perpendicular to the flow) but also moderately high spectral resolution to measure velocity differences on either side of a jet. Such requirements, at least at optical/UV wavelengths, are best met by the Space Telescope Imaging Spectrograph (STIS) on-board HST. Results suggest that YSO jets rotate with velocities that are consistent with them being launched at approximately $0.1-1.0 \mathrm{AU}$, i.e. values that are roughly in agreement with disk (D-) wind models. Further studies however are required to confirm this picture and new facilities, such as the Atacama Large Millimeter Array (ALMA), may well provide definitive observations in the future.

\section{Jets from more embedded sources}

Of considerable interest is the evolution of YSO jets with time. Up to now we have been discussing jets from so-called Class II YSOs, i.e. sources that are optically visible despite their large infra-red excess. These include not only the classical $\mathrm{T}$ Tauri stars but also their higher mass counterparts, the Herbig Ae/Be stars. What are the properties of jets at earlier phases, i.e. when the source is optically obscure? These stages are referred to as the Class 0 or Class I phases. Particularly in the case of Class 0 sources, not only the source but the outflow is optically embedded. Here one has to use infra-red (e.g. Spitzer/Herschel), mm (e.g. ALMA) and radio techniques to measure the outflow parameters. Such observations reveal a wealth of information. In particular (e.g. Dionatos et al. 2009; Podio \& Herschel GASPS Team 2011), we see that:

Infra-red spectra confirm that an underlying atomic jet is present.

The temperature and ionisation fraction of the flow is considerably lower than found in jets from Class I/II sources.

The amount of refractory species in gaseous form is very low indeed, i.e. most of these species are still locked up in dust grains. 
The mass loss rate in the "embedded" jet is typically $10-10^{2}$ times higher than corresponding jets from optically visible young stars.

\section{$5 \quad$ X-ray emission from YSO jets}

It has been known for many years that classical and weak-line $\mathrm{T}$ Tauri stars are sources of X-ray emission (e.g. Güdel \& Nazé 2009). While in the case of weak-line $\mathrm{T}$ Tauri stars this emission is thought to arise solely from chromospheric activity, jets certainly contribute in some classical T Tauri stars. An example is the recently conducted Chandra observations of DG Tau (Güdel et al. 2008). These show the central source has both a hard $(1.5-7.3 \mathrm{KeV})$ and a soft $(0.5-1.0 \mathrm{KeV})$ component. The hard component varies over a period of approximately a day whereas the soft component is much more steady. DG Tau also has a well-known optically visible jet which is seen on extended scales of hundreds of AU (Dougados et al. 2000). Long exposure Chandra images not only reveal this extended jet in X-rays but also show it has a soft spectrum reminiscent of the soft component at the source. This suggests the latter also arises from the jet. In any event we can estimate the temperature of the gas emitting the X-rays and typically this is around $4.10^{6} \mathrm{~K}$. Two other observations (Guedel et al. 2011) also support the idea that the soft component at the source is the jet:

An estimate of the neutral hydrogen column density, $\mathrm{N}_{\mathrm{H}}$, in front of the soft component, suggests a value 2-3 times smaller than expected based on the known extinction towards the source. This implies the soft component comes from a region beyond the centre.

PSF fitting to both the hard and soft components shows their centroids are offset. The displacement is only a fraction of an arcsecond, but interestingly it is in the direction of the extended jet.

Finally it is worth pointing out that the X-ray emission of a YSO jet is highly variable. In fact in the case of DG Tau, the counterjet was visible a number of years ago but has now faded out of sight (Guedel et al. 2011). This is consistent with the expected short cooling time of the high density X-ray emitting plasma.

\section{Radio emission from YSO jets}

Radio emission from YSO jets has been known for some time (e.g. Cohen \& Bieging 1986). It is typically found close to the source and often takes the form of an extension in the direction of the known outflow. Usually the observed spectral index implies the emission is thermal (bremsstrahlung) radiation and it is normally very weak. Thus observing jets in the radio band has been very challenging up to now; this situation however is about to change.

The use of fibre optic links means we will soon see an enormous improvement (by factors up to 20) in the sensitivity of radio interferometers (e.g. Dickman et al. 2010). Very faint continuum sources, such as jets from young stars, will 
be mapped in much more detail and new outflows will be discovered. This large increase in sensitivity will allow proper motion studies to be routinely carried out, at radio wavelengths, for the first time. Of equal importance is the fact that some YSO jets have non-thermal (i.e. synchrotron and gyro-synchrotron) radio emission as well as thermal emission. This is illustrated by recent observations of the powerful Herbig-Haro 80/81 outflow (Carrasco-González et al. 2010) which show its radio spectrum is inverted, i.e. it is non-thermal, and the emission is strongly polarised as expected from synchrotron radiation. Of particular note is the fact that the observed polarisation can be used to determine the direction of the outflow's magnetic field which, in this case, is found to be largely parallel to the outflow.

Thus observations at radio wavelengths have the potential to be very important to the study of YSO jets. This is because, as already pointed out, most jet parameters are well known from imaging and spectroscopy but the strength and the direction of the magnetic field has remained somewhat elusive. Assuming energy equipartition between the radiating electrons and the field, the latter's strength can be estimated in those cases were we observe synchrotron emission. We may thus soon be in a position where virtually all parameters in YSO jets can be prescribed with reasonable accuracy. Of course this gives us an excellent starting point not only for numerical simulations (see the contribution by Andrea Ciardi in this volume) but also, through the determination of the relevant dimensionless numbers, for laboratory jet simulations (see the contributions by Sergey Lebedev).

\section{References}

Antoniucci, S., Nisini, B., Giannini, T., \& Lorenzetti, D., 2008, A\&A, 479, 503

Arce, H.G., Borkin, M.A., Goodman, A.A., Pineda, J.E., \& Halle, M.W., 2010, ApJ, 715,1170

Bacciotti, F., Ray, T.P., Mundt, R., Eislöffel, J., \& Solf, J., 2002, ApJ, 576, 222

Bally, J., Reipurth, B., \& Davis, C.J., 2007, Protostars and Planets V, 215

Carrasco-González, C., Rodríguez, L.F., Anglada, G., et al., 2010, Science, 330, 1209

Codella, C., Cabrit, S., Gueth, F., et al., 2007, A\&A, 462, L53

Coffey, D., Bacciotti, F., Ray, T.P., Eislöffel, J., \& Woitas, J., 2007, ApJ, 663, 350

Cohen, M., \& Bieging, J.H., 1986, AJ, 92, 1396

Curtis, E.I., Richer, J.S., Swift, J.J., \& Williams, J.P., 2010, MNRAS, 408, 1516

Dickman, R., McKinnon, M., Chandler, C., et al., 2010, Soc. Photo Opt. Instrum. Eng. Conf. Ser., 7737

Dionatos, O., Nisini, B., Garcia Lopez, R., et al., 2009, ApJ, 692, 1

Dougados, C., Bacciotti, F., Cabrit, S., \& Nisini, B., 2010, Lecture Notes in Physics (Berlin Springer Verlag), 793, 213

Dougados, C., Cabrit, S., Lavalley, C., \& Ménard, F., 2000, A\&A, 357, L61

Dulwich, F., Worrall, D.M., Birkinshaw, M., Padgett, C.A., \& Perlman, E.S., 2007, MNRAS, 374, 1216

Ferreira, J., Dougados, C., \& Cabrit, S., 2006, A\&A, 453, 785 
Güdel, M., Skinner, S.L., Audard, M., Briggs, K.R., \& Cabrit, S., 2008, A\&A, 478, 797

Güdel, M., \& Nazé, Y., 2009, A\&A, 17, 309

Guedel, M., Audard, M., Bacciotti, F., et al., 2011 [ArXiv e-prints] [arXiv:1101.2780]

Hartigan, P., Frank, A., Foster, J.M., et al., 2011, ApJ, 736, 29

Livio, M., 1999, Phys. Rep., 311, 225

Livio, M., 2011, Am. Inst. Phys. Conf. Ser., 1358, 329

Marshall, H.L., Miller, B.P., Davis, D.S., et al., 2002, ApJ, 564, 683

O'Sullivan, S.P., \& Gabuzda, D.C., 2009, MNRAS, 400, 26

Piran, T., 2005, Nuovo Cimento C Geophysics Space Physics C, 28, 373

Podio, L., \& Herschel GASPS Team, 2011, IAU Symp., 280, 308P

Podio, L., Medves, S., Bacciotti, F., Eislöffel, J., \& Ray, T., 2009, A\&A, 506, 779

Pudritz, R.E., Ouyed, R., Fendt, C., \& Brandenburg, A., 2007, Protostars and Planets V, 277

Raga, A.C., de Colle, F., Kajdič, P., Esquivel, A., \& Cantó, J., 2007, A\&A, 465, 879

Ray, T., Dougados, C., Bacciotti, F., Eislöffel, J., \& Chrysostomou, A., 2007, Protostars and Planets V, 231

Sahai, R., 2002, Rev. Mex. Astron. Astrofis. Conf. Ser., 13, 133

Salmeron, R., Königl, A., \& Wardle, M., 2007, Ap\&SS, 311, 81

Seale, J.P., \& Looney, L.W., 2008, ApJ, 675, 427

Shu, F.H., Najita, J.R., Shang, H., \& Li, Z.-Y., 2000, Protostars and Planets IV, 789

Sikora, M., 2011, IAU Symp., 275, 59

Sparks, W.B., Biretta, J.A., \& Macchetto, F., 1996, ApJ, 473, 254

Whelan, E.T., Ray, T.P., Bacciotti, F., et al., 2005, Nature, 435, 652

Woitas, J., Bacciotti, F., Ray, T.P., et al., 2005, A\&A, 432, 149 